ARTIGO ORIGINAL

\title{
Volume total e número de repetições máximas durante séries de treinamento resistido: método tradicional vs. série agonista-antagonista
}

\author{
Total volume and maximum number of repetitions performed during resistance training sets: traditional \\ versus agonist-antagonist set methods
}

\author{
William Peneda Tozei, Milton Pereira Amaral, Washington Pires, \\ Renato Melo Ferreira, Everton Rocha Soares \\ Universidade Federal de Ouro Preto (UFOP), Ouro Preto/MG, Brasil
}

\section{HISTÓRICO DO ARTIGO \\ Recebido: 04 maio 2020 \\ Revisado: 25 junho 2020 \\ Aprovado: 31 julho 2020}

\section{PALAVRAS-CHAVE:}

Musculação; Métodos de Musculação; Volume Total.

\section{KEYWORDS:}

Resistance Training;

Methods of Resistance Training; Total Volume.

\section{RESUMO}

OBJETIVO: Comparar volume total e número repetições máximas (nRM) que pode ser realizado nos exercícios rosca direta (RD) e tríceps na polia (TP), variando a ordem de execução, nos métodos tradicional (TRAD) e série pareada agonista-antagonista (SPAA).

MÉTODOS: Doze indivíduos (22 $\pm 3,5$ anos) com experiência em treinamento resistido (TR), compareceram a 10 sessões com intervalo de 48 a 72 horas entre elas. Após avaliação antropométrica, três sessões de familiarização e realização de testes de uma repetição máxima (1-RM) e reprodutibilidade de 1-RM, foram realizados quatro protocolos experimentais para mensuração do nRM, utilizando 70\% de 1-RM: TRAD1 (quatro séries no exercício RD + quatro séries no exercício TP); TRAD2 (quatro séries no exercício TP + quatro séries no exercício RD); SPAA1 (quatro séries no exercício RD e TP) e; SPAA2 (quatro séries no exercício TP e RD). Sob o método TRAD, foram implementados intervalos de 90 segundos entre todas séries realizadas; um intervalo de descanso de dois minutos foi implementado entre a conclusão da última série do primeiro exercício e a primeira série do segundo exercício (RD + TP ou TP + RD). Sob o método SPAA, nenhum intervalo foi implementado entre o primeiro e segundo exercício (RD e TP ou TP e RD); noventa segundos de intervalo de descanso foram implementados após conclusão do primeiro e do exercício subsequente.

RESULTADOS: Não foram observadas diferenças no volume total e percepção subjetiva do esforço (PSE) entre os protocolos experimentais, enquanto foi verificado maior nRM no exercício TP em comparação com o RD em todos os protocolos utilizados.

CONCLUSÃO: Na intensidade de 70\% de 1-RM, o volume total não é influenciado pelo método de TR (TRAD ou SPAA) e pela ordem dos exercícios (RD e TP ou TP e RD). Adicionalmente, observou-se que o nRM parece ser influenciado pelo grupamento muscular utilizado.

\section{ABSTRACT}

OBJECTIVE: To compare the total volume and number of maximum repetitions ( $\mathrm{nMR}$ ) that can be performed in barbell curl (BC) and triceps pushdown (TP) exercises, varying order of execution, on traditional (TRAD) and agonist-antagonist paired set (AAPS) methods.

METHODS: Twelve individuals ( $22 \pm 3.5$ years) with experience in resistance training (RT), attended 10 sessions with an interval of 48 to 72 hours between them. After anthropometric evaluation, three familiarization sessions, one repetition maximum tests (1-RM) and reproducibility, four experimental protocols were performed to measure the total volume and $n M R$, using $70 \%$ of 1-RM: TRAD1 (four sets of exercise BC + four sets of exercise TP); TRAD2 (four sets of TP exercise + four sets of BC exercise); AAPS1 (four sets of exercise BC and TP) and; AAPS2 (four sets of exercise TP and BC). Under the TRAD method, 90-seconds rest intervals were implemented between all sets; two-minute rest interval between the completion the last set of first exercise and the first set of second exercise was implemented (BC + TP or TP + BC). Under the AAPS method, no interval was implemented between first and second exercise (BC and TP or TP and BC); a 90-seconds rest interval after the completion of the first and the subsequent exercise was implemented.

RESULTS: There were no differences in total volume and rating of perceived exertion (RPE) between the experimental protocols, while a higher $\mathrm{nMR}$ was found in the TP exercise compared to the BC in all protocols used.

CONCLUSION: It is concluded that in the intensity of 70\% 1-RM, the total volume is not influenced by the TR method (TRAD or AAPS) and by the order of the exercises (BC and TP or TP and BC). Additionally, it was observed that the nMR seems to be influenced by the muscle group used. 


\section{INTRODUÇÃO}

O treinamento resistido (TR) com objetivo de hipertrofia muscular tem sido empregado em diferentes populações (SHEPSTONE et al., 2005; BURESH et al., 2009; SCHOENFELD et al., 2016; NÚÑEZ et al., 2018). No entanto, o impacto na hipertrofia muscular induzido pelo TR depende da estruturação e interação de diferentes componentes de carga como intensidade, volume, intervalo entre séries e ordem dos exercícios (BOTTARO et al., 2007; SCHOENFELD, 2010; SCHOENFELD et al., 2016; SCHOENFELD et al., 2019).

A intensidade no TR pode ser empregada pelo uso de quilagens relativas à máxima capacidade de produzir força (conhecida também como uma repetição máxima ou 1-RM) e pelo número de repetições máximas (nRM) que pode ser realizado nos exercícios resistidos. De forma geral, a utilização de intensidades entre $67 \%$ a $85 \%$ de 1-RM e 6 a 12-RM são eficientes para indução de hipertrofia muscular (ACSM, 2009; SCHOENFELD, 2010). No entanto, outros estudos mostraram que quando os exercícios de TR são realizados até a fadiga voluntária a utilização de cargas entre $30-60 \%$ de 1-RM resulta em hipertrofia muscular semelhante ao TR com cargas maiores do que $60 \%$ de 1-RM (OGASAWARA et al., 2013; SCHOENFELD et al., 2015). Embora o nRM esteja associado com a intensidade (BACHLE; EARLE, 2010; SCHOENFELD, 2010), o nRM que pode ser executado com intensidades relativas de 1-RM apresenta grande variação em função de fatores como a ordem de execução dos exercícios (BALSAMO et al., 2013) e o método de TR escolhido (WEAKLEY et al., 2017) .

Ao considerar a influência que a ordem dos exercícios resistidos tem sobre nRM, Miranda et al. (2013) afirmam que é possível realizar maior nRM alterando a disposição dos exercícios realizados, no início ou final da sessão. No referido estudo ao investigar se a randomização na ordem dos exercícios supino, desenvolvimento de ombros e extensão de tríceps, em uma sessão de TR, induziria diferença no nRM, foi observado, de forma geral, que quando um determinado exercício é realizado no início da sessão é possível realizar um maior nRM no mesmo.

Já ao considerar a influência de um método de TR sobre o nRM, deve-se levar em consideração as características desse quanto a estruturação dos componentes de carga de treino, uma vez que manipulação da intensidade influi sobre a quantidade de tensão mecânica e dano muscular e a manipulação do volume (como o número se séries e repetições) e intervalo entre séries influem sobre a quantidade de estresse metabólico produzido (SCHOENFELD, 2010).

Dentre os diferentes métodos de TR existentes tem-se o método tradicional (TRAD) e o da série pareada agonistaantagonista (SPAA). O método TRAD é caracterizado pela realização de um determinado número de séries em um mesmo exercício, com intervalo de recuperação entre elas (PAZ et al., 2017). Já o método SPAA consiste em realizar séries alternadas de dois exercícios, sem intervalo entre elas, para grupos musculares agonistas e antagonistas em uma articulação (PAZ et al., 2017). Embora os métodos TRAD e SPAA apresentem características próprias quanto a estruturação da ordem de exercícios e intervalo entre séries, ainda não existe consenso se há ou não diferença entre o nRM que pode ser realizado em cada um desses métodos, para uma mesma intensidade
(PAZ et al., 2017; SOUZA et al., 2017). Souza et al. (2017) não observaram diferença no nRM entre os métodos TRAD e SPAA, nas três séries do exercício supino e remada sentada. Por outro lado, Paz et al. (2017) observaram maior nRM e volume total (número de séries $x$ número de repetições $x$ peso levantado) no método SPAA quando comparado com o TRAD, em cada uma das três séries do exercício supino e remada sentada.

Portanto, o objetivo foi comparar o volume total e nRM que pode ser realizado nos exercícios rosca direta (RD) e tríceps na polia (TP), variando a ordem de execução, nos métodos TRAD e SPAA.

\section{MÉTODOS}

Participaram do estudo doze homens $(22,9 \pm 3,5$ anos; $78,6 \pm 7,1 \mathrm{~kg} ; 175,8 \pm 7,0 \mathrm{~cm}$ e; $11,0 \pm 3,9 \% \mathrm{GC}$ ). Os critérios de inclusão foram: a) ter entre 18 e 30 anos; b) ter ao menos seis meses de experiência em TR e ter os exercícios TP e RD incorporados a rotina de treino; $c$ ) não indicação de resposta positiva no questionário PAR-Q (THOMPSON et al., 2013); e d) não apresentar nenhum problema osteomioarticular.

Os critérios de exclusão foram: a) fazer uso de suplemento ou alimento (como creatina, cafeína, bebida alcoólica, etc.) que pudesse influir sobre a força muscular, nas 48 horas que antecederam a coleta e; b) fazer uso de esteroides anabolizantes; Todos participantes foram orientados a não realizar nenhum tipo de exercício físico nas 48 horas antes dos testes e sessões experimentais. Este estudo foi aprovado pelo Comitê de Ética em Pesquisa da Universidade Federal de Ouro Preto (UFOP), sob Parecer $n^{\circ}$. 1.830.603.

\section{A rotina estabelecida para a coleta foi:}

I) Avaliação antropométrica e sessões de familiarização: Após avaliação antropométrica três sessões de familiarização foram realizadas com intervalo de 48 a 72 horas entre elas, com o objetivo de padronizar a execução dos exercícios (ritmo e amplitude); foram realizadas quatro séries de 10 repetições, nos exercício RD e TP, no ritmo 1:2 (um segundo na ação concêntrica e dois segundos na ação excêntrica), com percepção de esforço (PSE) entre os números cinco e seis da escala de OMNI-RES (ROBERTSON et al., 2003);

II) Predição de 1-RM: Entre 48 e 72 horas da última sessão de familiarização foram realizados randomicamente testes de predição de 1-RM (BRZYCKI, 1993) para os exercícios RD e TP;

III) Testes de 1-RM: Entre 48 e 72 horas da sessão de predição de 1-RM foi realizado randomicamente o teste de 1-RM, conforme descrito por Baechle; Earle (2010);

IV) Reprodutibilidade dos testes de 1-RM: Entre 48 e 72 horas dos testes de 1-RM, foi avaliada a reprodutibilidade dos mesmos (BAECHLE; EARLE, 2010);

V) Protocolos experimentais: Entre 48 e 72 horas após os testes de reprodutibilidade foram avaliados volume total e nRM nos exercícios RD e TP. A intensidade foi de $70 \%$ de 1-RM e o ritmo 1:2. Foram quatro protocolos experimentais que variaram quanto a ordem de execução dos exercícios e intervalo, de acordo com o método utilizado (TRAD ou SPAA).

Um intervalo de 48 e 72 horas foi adotado entre cada protocolo experimental. 
Uma série de 10 a 15 repetições, com 40\% 1-RM, nos exercícios RD e TP foi realizada como forma de aquecimento antes de cada sessão experimental. Três minutos após o aquecimento os protocolos experimentais foram randomizados em cada sessão, sendo:

1) Tradicional 1 (TRAD1): RD + TP. Para o exercício RD foram quatro séries de RM com 90 segundos de intervalo entre as séries e; após intervalo de 120 segundos, foi realizado o exercício TP, também em quatro séries de RM e 90 segundos de intervalo entre séries;

2) Tradicional 2 (TRAD2): Semelhante ao TRAD1, porém alterando a ordem dos exercícios, iniciando com TP;

3) Série pareada agonista-antagonista 1 (SPAA1): Aglutinação de dois exercícios realizados em sequência, sem intervalo entre eles. Iniciou-se pela execução do exercício RD e, sem intervalo, executou a o TP. Após a realização dos dois exercícios realizava-se intervalo de 90 segundos, repetindo o processo por mais três vezes (total de quatro séries);

4) Série pareada agonista-antagonista 2 (SPAA2): Semelhante ao SPAA1, porém alterando a ordem dos exercícios, iniciando com TP.

Imediatamente após o final de cada série, os voluntários foram instruídos a apontar uma nota na escala de OMINI-RES (ROBERTSON et al., 2003).

Para os protocolos TRAD (1 e 2), foram coletados oito valores e nos protocolos SPAA (1 e 2 ) foram coletados quatro valores de PSE. Devido à diferença entre o número de valores de PSE coletados nos protocolos (TRAD e SPAA), a avaliação de possíveis diferenças nos valores desta variável foi a partir da média aritmética dos resultados obtidos em cada série. Por fim, interrupção ocorreria caso o voluntário não realizasse a amplitude total de movimento e não se mantivesse dentro do ritmo de execução proposto. Para além, foi solicitado que os mesmos não realizassem outros exercícios físicos no período de até 48 horas antes de cada protocolo. Destaca-se que, em todos os protocolos, houve estímulo verbal (motivação extrínseca) a todos os voluntários. $\mathrm{O}$ estudo foi conduzido por dois avaliadores experientes.

Os dados estão apresentados em média \pm desvio padrão. Para avaliação da distribuição de normalidade foi utilizado o teste de D’agostino e Pearson. Para as comparações foram utilizados os seguintes testes: a) Reprodutibilidade entre os testes de 1-RM: teste t de Student; b) Entre os valores do volume total nos diferentes protocolos experimentais: ANOVA two way $(2 \mathrm{x}$ 4) para medidas repetidas e pós-teste de Bonferroni; c) Entre os valores de PSE nos diferentes protocolos de treinamento: ANOVA one way para medidas repetidas; d) Entre o nRM realizado entre as quatro séries de cada exercício nos respectivos protocolos: ANOVA one way para medidas repetidas e pós-teste de Newman-Keuls ou Dunns, quando necessário. A análise estatística foi feita no Software Graphpad Prism (versão 5.00), com nível de significância de $p<0,05$.

Tabela 1. Número de repetições máximas (nRM) nas quatro séries dos exercícios RD e TP nos quatro protocolos experimentais.

\begin{tabular}{lcccccccc}
\hline \multirow{2}{*}{ Repetições } & \multicolumn{4}{c}{ nRM - RD } & \multicolumn{5}{c}{ nRM - TP } \\
\cline { 2 - 8 } & TRAD1 & TRAD2 & SPAA1 & SPAA2 & TRAD1 & TRAD2 & SPAA1 & SPAA2 \\
\hline Mínimo & 20,0 & 18,0 & 19,0 & 17,0 & 29,0 & 27,0 & 28,0 & 30,0 \\
Máximo & 34,0 & 30,0 & 33,0 & 32,0 & 40,0 & 46,0 & 45,0 & 45,0 \\
Média & 26,9 & 24,3 & 27,1 & 24,8 & $36,0^{*}$ & $37,8^{*}$ & $36,3^{*}$ & $38,7^{*}$ \\
DP & 5,0 & 4,0 & 4,5 & 4,7 & 3,8 & 7,1 & 4,7 & 4,7 \\
\hline
\end{tabular}

Nota: TRAD1 = Método Tradicional 1 (RD com intervalo de 120s e TP); TRAD2 = Método Tradicional 2 (TP com intervalo de 120s e RD); SPAA1 = Método série pareada agonista-antagonista 1 (RD seguido de TP sem intervalo); SSAA2 = Método série pareada agonista-antagonista 2 (TP seguido de RD sem intervalo); RD = Rosca direta; TP = Tríceps na polia; DP = Desvio padrão.

* $p<0,05$ comparado com o exercício RD (Anova two-way seguido pelo teste de Bonferroni).

\section{RESULTADOS}

Observou-se reprodutibilidade $(p>0,05)$ entre os testes de 1-RM (BAECHLE; EARLE, 2010) para os exercícios RD e TP (RD: $48,0 \pm 6,1 \mathrm{~kg}$ para $1-\mathrm{RM}$ e $47,8 \pm 7,0 \mathrm{~kg}$ para a reprodutibilidade e; TP: $85,1 \pm 7,6 \mathrm{~kg}$ para $1-\mathrm{RM}$ e $85,1 \pm 7,1 \mathrm{~kg}$ para a reprodutibilidade).

Ao analisar, o volume total realizado ( $\mathrm{nRM} x$ número de séries $x$ quilagem levantada, em quilogramas) em cada protocolo experimental, por meio da Anova two-way de medidas repetidas, não se observou diferenças significativas entre os quatro protocolos experimentais (TRAD1: $3.007 \pm 451 \mathrm{~kg}$, vs. TRAD2: $3.014 \pm 542 \mathrm{~kg}$, vs. SPAA1: $3.037 \pm 501 \mathrm{~kg}$, vs. SPAA2: $3.127 \pm 506 \mathrm{~kg}$ ).

Ao avaliar a interação entre o nRM que pode ser realizado nos 4 protocolos para cada um dos exercícios (RD e TP), não foram observadas interações (Tabela 1). Contudo, destaca-se que o $n R M$ realizado no TP foi maior $(p<0.05)$ do que no RD independentemente do método de TR utilizado (TRAD ou SPAA).
A Tabela 2 apresenta valores médios e desvio padrão do nRM para cada uma das quatro séries dos exercícios RD e TP nos quatro protocolos realizados. De forma geral, observou-se que independentemente do exercício físico e do protocolo experimental utilizado, o nRM realizado na terceira e quarta série foi menor do que o da primeira. No entanto, não foram observadas diferenças entre o nRM realizado entre a segunda e terceira série no exercício RD nos protocolos TRAD2 e SPAA1, diferentemente do observado para o mesmo exercício nos demais protocolos e para o TP nos quatro protocolos.

Adicionalmente, no exercício RD do protocolo TRAD2 não foram observadas diferenças entre o nRM realizado entre a segunda e primeira série e entre a quarta e segunda série, diferentemente do observado para o mesmo exercício nos demais protocolos e para o TP nos quatro protocolos. Em relação aos valores relativos a percepção de esforço (OMNI-RES; ROBERTSON et al., 2003), a partir da anova one-way para medidas repetidas, não foram observadas diferença entre as sessões ex- 
Tabela 2. Número de repetições máximas (nRM) em cada série dos exercícios RD e TP nos quatro protocolos experimentais.

\begin{tabular}{ccccccccc}
\hline \multirow{2}{*}{ Série } & \multicolumn{2}{c}{ TRAD1 } & \multicolumn{2}{c}{ TRAD2 } & \multicolumn{2}{c}{ SPAA1 } & \multicolumn{2}{c}{ SPAA2 } \\
\cline { 2 - 8 } & RD & TP & TP & RD & RD & TP & TP & RD \\
\hline $1^{\text {a }}$ & $10,1 \pm 2,8$ & $13,1 \pm 1,7$ & $12,9 \pm 2,2$ & $9,2 \pm 3,0$ & $10,1 \pm 2,5$ & $12,8 \pm 1,9$ & $13,3 \pm 2,1$ & $8,6 \pm 2,2$ \\
$2^{\text {a }}$ & $6,8 \pm 1,4^{\mathrm{a}}$ & $8,9 \pm 0,7^{\mathrm{a}}$ & $9,1 \pm 2,2^{\mathrm{a}}$ & $6,6 \pm 1,7$ & $6,7 \pm 1,4^{\mathrm{a}}$ & $9,3 \pm 1,4^{\mathrm{a}}$ & $9,9 \pm 1,3^{\mathrm{a}}$ & $6,6 \pm 1,0^{\mathrm{a}}$ \\
$3^{3}$ a & $5,3 \pm 0,9^{\mathrm{a}, \mathrm{b}}$ & $7,2 \pm 1,3^{\mathrm{a}, \mathrm{b}}$ & $7,5 \pm 1,6^{\mathrm{a}, \mathrm{b}}$ & $5,3 \pm 2,0^{\mathrm{a}}$ & $5,8 \pm 0,9^{\mathrm{a}, \mathrm{b}}$ & $7,5 \pm 1,2^{\mathrm{a}, \mathrm{b}}$ & $7,9 \pm 1,4^{\mathrm{a}, \mathrm{b}}$ & $5,2 \pm 1,0^{\mathrm{a}, \mathrm{b}}$ \\
$4^{\text {a }}$ & $4,7 \pm 1,1^{\mathrm{a}, \mathrm{b}}$ & $6,7 \pm 1,5^{\mathrm{a}, \mathrm{b}}$ & $6,9 \pm 2,0^{\mathrm{a}, \mathrm{b}}$ & $4,6 \pm 1,7^{\mathrm{a}}$ & $4,5 \pm 0,7^{\mathrm{a}, \mathrm{b}, \mathrm{c}}$ & $6,8 \pm 1,6^{\mathrm{a}, \mathrm{b}}$ & $7,5 \pm 1,3^{\mathrm{a}, \mathrm{b}}$ & $4,5 \pm 1,3^{\mathrm{a}, \mathrm{b}}$ \\
\hline
\end{tabular}

Nota: TRAD1 = Método Tradicional 1 (RD com intervalo de 120s e TP); TRAD2 = Método Tradicional 2 (TP com intervalo de 120s e RD); SPAA1 = Método série pareada agonista-antagonista 1 (RD seguido de TP sem intervalo); SSAA2 = Método série pareada agonistaantagonista 2 (TP seguido de RD sem intervalo); $\mathrm{RD}=$ Rosca direta; $\mathrm{TP}=$ Tríceps na polia

${ }_{\mathrm{b}}^{\mathrm{a}} \mathrm{p}<0,05$ comparado com a 1 a série do mesmo exercício;

'p<0,05 comparado com a 3a série do mesmo exercício (Anova one-way seguido pelo teste de Newman-Keuls ou Dunns, quando necessário).

perimentais (TRAD1: 7,8 $\pm 0,0$ vs. TRAD2: $=7,9 \pm 1,0$ vs. SPAA1: $7,9 \pm 0,7$ vs. SPAA2: $8,0 \pm 0,7)$.

O objetivo do presente estudo foi comparar o volume total e $n R M$ que pode ser realizado até a fadiga nos exercícios RD e TP, variando a ordem de execução, nos métodos TRAD e SPAA. De forma geral, os resultados não mostraram diferença entre os valores de volume total dos exercícios RD e TP, nos diferentes protocolos (TRAD1 vs. TRAD2 vs. SPAA1 vs. SPAA2). Contudo, para todos os protocolos, o nRM no TP foi maior que no RD.

$\mathrm{O}$ volume total é caracterizado pelo nRM $\mathrm{x}$ número de séries $x$ peso levantado $(\mathrm{kg})$, sendo influenciado por fatores como intensidade, intervalo de recuperação e ritmo de execução (WILLARDSON; BURKETT, 2005; RICHENS; CLEATHER, 2014). A partir disso, acredita-se que a estruturação metodológica adotada para os quatro protocolos (intensidade de $70 \%$ de 1-RM, ritmo 1:2 e intervalo entre séries de 90 segundos) influenciou sobremaneira a não ocorrência de diferenças entre o volume total dos quatro protocolos experimentais. Por outro lado, Paz et al. (2017) observaram maior volume total no método SPAA do que no TRAD. Destaca-se que no estudo de Paz et al. (2017) os exercícios investigados foram supino e remada sentada, a intensidade igual a 10-RM e o intervalo entre séries de 120 segundos. Em conjunto, esses dados reforçam a ideia que a grande possibilidade de estruturação do TR, quanto os tipos de exercícios, intensidade e intervalo entre séries, por exemplo, dificulta a comparação dos resultados de diferentes estudos. Assim, são necessários estudos que se proponham comparar o efeito de diferentes estruturações de intensidade, ritmo de execução e intervalo entre séries sobre o volume total de treinamento em diferentes métodos de TR.

Ao considerar o componente de carga ordem dos exercícios, estudos observaram que a manipulação desse componente no TR induzem diferenças no nRM que pode ser realizado (BALSAMO et al., 2012; BALSAMO et al., 2013; MIRANDA et al., 2013); no entanto, pouco se investigou sobre a ordem dos exercícios utilizando o método SPAA (BALSAMO et al., 2012). A partir dos resultados obtidos no presente estudo, observa-se que, independentemente do método de TR utilizado e ordem dos exercícios, o nRM não difere. Esse resultado, em parte, é explicado pelo número de exercícios utilizados (apenas dois, RD e TP) e pelos grupamentos musculares envolvidos, uma vez que, em outros estudos, quando há influência da ordem dos exercícios no nRM, os grupamentos musculares e o número de exercícios são diferentes dos utilizados no presente trabalho (BALSAMO et al., 2012; BALSAMO et al., 2013; MIRANDA et al., 2013).

No tocante ao $\mathrm{nRM}$, foram observados maiores valores no TP em comparação ao RD (Tabela 1). Acredita-se que este resultado tenha sido influenciado pelo maior volume e área de secção transversa (AST) das três porções do músculo tríceps braquial em comparação com os flexores do cotovelo (AN et al., 1981; PETERSON; RAYAN, 2011), já que existe a possibilidade de realizar maior nRM em exercícios envolvendo maior massa muscular (CHAGAS; VARGAS; LIMA, 2005; GROSICKI; MILLER; MARSH, 2014) uma vez que a AST possui forte correlação positiva com a força muscular (MAUGHAN; WATSON; WEIR, 1983).

No presente estudo, o método de TR e a ordem de execução dos exercícios não influenciaram a somatória do nRM nas quatro séries de RD e TP. No entanto, observa-se comportamento diverso no decaimento do nRM nas séries subsequentes de cada exercício nos diferentes protocolos, uma vez que nem sempre ocorreu redução significativa entre o nRM realizado em uma determinada série e a precedente, sobretudo no exercício RD. Em parte, acredita-se que a utilização de intensidade (70\% de 1-RM) e intervalo entre séries moderados ( 90 segundos) (SCHOENFELD, 2010) tenha alguma influência nesse resultado. Uma vez que a utilização de cargas submáximas e intervalos maiores do que 60 segundos, favorecem a manutenção da performance entre as séries (LIMA et al., 2006; GRGIC et al., 2017; GRGIC et al., 2018). Adicionalmente, embora todos os voluntários tinham no mínimo seis meses de experiência com TR, somado ao número de voluntários do estudo, deve-se considerar a possibilidade de existência de diferenças individuais quanto a resistência de força muscular, o que pode impactar o desempenho nos exercícios, uma vez que indivíduos mais treinados tendem a conseguir realizar um maior volume total do que aqueles menos treinados, para uma mesma intensidade relativa (SCHOENFELD, 2010). Cabe lembrar que não avaliamos a resistência de força muscular dos voluntários, o que perfaz uma limitação desse estudo. Mais estudos precisam ser realizados para compreender melhor esses resultados.

A escala de OMNI-RES é utilizada como um instrumento que possibilita controlar e verificar a intensidade do TR (NACLERIO et al., 2011; MIRANDA et al., 2013); ainda assim, não foram encontradas diferenças significativas para PSE entre os protocolos (TRAD1, TRAD2, SPAA1 e SPAA2). Isto é, em parte, explicado pela não existência de diferenças no volume total entre os diferentes protocolos. Tais resultados corroboram Silva et al. (2009), que não identificaram diferenças da PSE entre diferentes proto- 
colos de TR, porém, com mesmo volume total de treinamento. Além disso, outros aspectos que podem ter influenciado estão relacionados à utilização da mesma intensidade (70 \% de 1-RM) em todos os protocolos e a orientação aos voluntários para realizarem nRM. Adicionalmente, embora os resultados não demonstrem diferenças entre o volume total e PSE nos protocolos experimentais utilizados, podemos destacar como limitação do estudo a utilização de apenas dois exercícios (RD e TP) e não ter avaliado o lactato sanguíneo, uma vez que Souza et a. (2017) observaram maiores valores de PSE e lactato sanguíneo no método SPAA em relação ao TRAD.

Por fim, salienta-se que a avaliação da força máxima foi determinada a partir do teste de 1-RM (BAECHLE; EARLE, 2010), teste considerado como padrão-ouro (VERDIJK et al., 2009). Assim a verificação de reprodutibilidade nos testes de 1-RM demonstra consistência nos valores de força máxima encontrados.

Sugerimos que novos estudos que busquem avaliar o efeito de diferentes métodos de treinamento e ordem de exercício sobre o volume total devem ser realizados com o intuito de utilizar um número maior de exercícios, analisando a adaptações na força e hipertrofia muscular bem como respostas metabólicas ao exercício.

\section{CONCLUSÃO}

Conclui-se que na intensidade de 70\% 1-RM, o volume total não é influenciado pelo método de TR (TRAD e SPAA) e ordem dos exercícios (RD e TP ou TP e RD), o que favorece, no aspecto otimização do tempo da sessão de TR, a utilização do método SPAA. O nRM que pode ser executado parece ser influenciado pelo grupamento muscular utilizado. Adicionalmente, pode-se considerar que apesar de ser postulado relação entre as intensidades 6 a 12-RM e $67 \%$ a $85 \%$ de 1-RM para hipertrofia muscular, foi possível verificar que na intensidade de 70\% de 1-RM, o $n R M$ variou além dessa faixa de RM.

\section{REFERÊNCIAS}

ACSM. American College of Sports Medicine. American College of Sports Medicine position stand. Progression models in resistance training for healthy adults. Medicine and Science in Sports and Exercise, Madison, v. 41, n. 3, p. 687-708, 2009.

AN, K. N.; HUI, F. C.; MORREY, B. F.; LINSCHEID, R. L.; CHAO, E. Y. Muscles across the elbow joint: a biomechanical analysis. Journal of Biomechanics, Elmsford, v. 14, n. 10, p. 659-69, 1981.

BAECHLE, T. R.; EARLE, R. W. Fundamentos do treinamento de força e do condicionamento. 3. ed. Barueri: Manole, 2010.

BALSAMO, S.; TIBANA, R. A.; NASCIMENTO, D. D. A. C.; FARIAS, G. L.; PETROCCELLI, Z.; SANTANA, F. S.; MARTINS, O. V.; AGUIAR, F.; PEREIRA, G. B.; SOUZA, J. C.; PRESTES, J. Exercise order affects the total training volume and the ratings of perceived exertion in response to a super-set resistance training session. International Journal of General Medicine, Macclesfield, v. 5, p. 123-7, 2012.

BALSAMO, S.; TIBANA. R. A.; NASCIMENTO, D. D. A. C.; FRANZ, C. B.; LYONS, S.; FAIGENBAUM, A.; PRESTES, J. Exercise order influences number of repetitions and lactate levels but not perceived exertion during resistance exercise in adolescents. Research in Sports Medicine, Philadelphia, v. 21, n. 4, p. 293-304, 2013.

BOTTARO, M.; MACHADO, S. N.; NOGUEIRA, W.; SCALES, R.; VELOSO, J. Effect of high versus low-velocity resistance training on muscular fitness and functional performance in older men. European Journal of Applied Physiology, Berlin, v. 99, n. 3, p. 257-64, 2007

BRZYCKI, M. Strength testing-predicting a one-rep max from reps-to-fatigue. Journal of Physical Education, Recreation \& Dance, v. 64, n. 1, p. 88-90, 1993.
BURESH, R.; BERG, K.; FRENCH J. The effect of resistive exercise rest interval on hormonal response, strength, and hypertrophy with training. Journal of Strength \& Conditioning Research, Lincoln, v. 23, n. 1, p. 62-71, 2009.

CHAGAS, M. H.; BARBOSA, J. R. M.; LIMA, F. V. Comparação do número máximo de repetições realizadas a 40 e $80 \%$ de uma repetição máxima em dois diferentes exercícios na musculação entre os gêneros masculino e feminino. Revista Brasileira de Educação Física e Esporte, São Paulo, v. 19, n. 1, p. 5-12, 2005.

GRGIC, J.; LAZINICA, B. MIKULIC, P. KRIEGER, J. W.; SCHOENFELD, B. J. The effects of short versus long inter-set rest intervals in resistance training on measures of muscle hypertrophy: a systematic review. European Journal of Sport Science, Berlin, v. 17, n. 8, p. 983-93, 2017.

GRGIC, J.; SCHOENFELD, B. J.; SKREPNIK, M.; DAVIES, T. B.; MIKULIC, P. Effects of rest interval duration in resistance training on measures of muscular strength. Sports Medicine, Auckland, v. 48, n. 1, p. 137-51, 2018

GROSICKI, G. J.; MILLER, M. E.; MARSH, A. P. Resistance exercise performance variability at submaximal intensities in older and younger adults. Clinical Interventions in Aging, Auckland, v. 9, p. 209-18, 2014.

LIMA, F. V.; CHAGAS, M. H.; CORRADI, E. F. F.; SILVA, G. F. Analysis of two training programs with different rest periods between series based on guidelines for muscle hypertrophy in trained individuals. Revista Brasileira de Medicina do Esporte, São Paulo, v. 12, n. 4, p. 175-8, 2006.

MAUGHAN, R. J.; WATSON, J. S.; WEIR, J. Strength and cross-sectional area of human skeletal muscle. Journal Physiology, London, v. 338, p. 37-49, 1983.

MIRANDA, H.; FIGUEIREDO, T.; RODRIGUES, B.; PAZ, G. A.; SIMÃO R. Influence of exercise order on repetition performance among all possible combinations on resistance training. Research in Sports Medicine, Philadelphia, v. 21, n. 4, p. 355-66, 2013

NACLERIO, F.; RODRÍGUEZ-ROMO, G.; BARRIOPEDRO-MORO, M. I.; JIMÉNEZ, A.; ALVAR, B. A.; TRIPLETT, N. T. Control of resistance training intensity by the OMNI perceived exertion scale. Journal of Strength \& Conditioning Research, Lincoln, v. 25, n. 7, p. 1879-88, 2011.

NÚÑEZ, F. J.; SANTALLA, A.; CARRASQUILA, I.; ASIAN, J. A.; REINA, J. I.; SUAREZ-ARRONES, L. J. The effects of unilateral and bilateral eccentric overload training on hypertrophy, muscle power and COD performance, and its determinants, in team sport players. PLoS One, San Francisco, v. 13, n. 3, p. 1-13, 2018.

OGASAWARA, R.; LOENNEKE, J. P.; THIEBAUD, R. S.; ABE, T. Low-load bench press training to fatigue results in muscle hypertrophy similar to high-load bench press training. International Journal of Clinical Medicine, v. 4, n. 2, p. $114-21,2013$

PAZ, G. A.; ROBBINS, D. W.; OLIVEIRA, C. G.; BOTTARO, M.; MIRANDA, H. Volume load and neuromuscular fatigue during an acute bout of agonist-antagonist paired-set vs. traditional-set training. The Journal of Strength \& Conditioning Research, Lincoln, v. 31, n. 10, p. 2777-84, 2017.

PETERSON, S. L.; RAYAN, G. M. Shoulder and upper arm muscle architecture. Journal of Hand Surgery, Oxford, v. 36, n. 5, p. 881-9, 2011.

RICHENS, B.; CLEATHER, D. J. The relationship between the number of repetitions performed at given intensities is different in endurance and strength trained athletes. Biology of Sport, Warsaw, v. 31, n. 2, p. 157-61, 2014.

ROBERTSON, R. J.; GOSS, F. L.; RUTKOWSKI, J.; LENZ, B.; DIXON, C.; TIMMER, J.; FRAZEE, K.; BUBE, J.; ANDREACCI, J. Concurrent validation of the OMNI perceived exertion scale for resistance exercise. Medicine \& Science in Sports \& Exercise, Madison, v. 35, n. 2, p. 333-41, 2003.

SCHOENFELD, B. J.; CONTRERAS, B.; KRIEGER, J.; GRGIC, J.; DELCASTILLO, K.; BELLIARD, R.; ALTO, A. Resistance training volume enhances muscle hypertrophy but not strength in trained men. Medicine \& Science and Sports \& Exercise, Madison, v. 51, n. 1, p. 94-103, 2019.

SCHOENFELD, B. J.; PETERSON, M. D.; OGBORN, D.; CONTRERAS, B.; SONMEZ, G. T. Effects of low- vs. high-load resistance training on muscle strength and hypertrophy in well-trained men. The Journal of Strength \& Conditioning Research, Lincoln, v. 29, n. 10, p. 2954-63, 2015.

SCHOENFELD, B. J.; OGBOR, D.; KRIEGER, J. W. Effects of Resistance Training Frequency on Measures of Muscle Hypertrophy: A Systematic Review and Meta-Analysis. Sports Medicine, Auckland, v. 46, n. 11, p. 1689-97, 2016. 
SCHOENFELD, B. J. The mechanisms of muscle hypertrophy and their application to resistance training. The Journal of Strength \& Conditioning Research, Lincoln, v. 24, n. 10, p. 2857-72, 2010.

SHEPSTONE, T. N.; TANG, J. E.; DALLAIRE, S.; SCHUENKE, M. D.; STARON, R. S.; PHILLIPS, S. M. Short-term high- vs. low-velocity isokinetic lengthening training results in greater hypertrophy of the elbow flexors in young men. Journal of Applied Physiology, Washington, v. 98, n. 5, p. 1768-76, 2005.

SILVA, R. P.; NOVAES, J. S.; AQUINO, M. S.; BOTTARO, M. Protocolos de treinamento resistido de alta velocidade de contração muscular em idosas: efeitos na percepção de esforço. Revista da Educação Física, Maringá, v. 20, n. 1, p. 77-84, 2009.

SOUZA, J. A. A. A.; PAZ, G. A.; MIRANDA, H. Blood lactate concentration and strength performance between agonist-antagonist paired set, superset and traditional set training. Archivos de Medicina del Deporte, Pamplona, v. 34, n. 3, p. 145-150, 2017.

THOMPSON, P. D.; ARENA, R.; RIEBE, D.; PESCATELLO, L. S. ACSM's new preparticipation health screening recommendations from ACSM's guidelines for exercise testing and prescription. Current Sports Medicine Reports, Philadelphia, v. 12, n. 4, p. 215-7, 2013.

VERDIJK, L. B.; VAN LOON, L.; MEIJER, K.; SAVELBERG, H. H. One-repetition maximum strength test represents a valid means to assess leg strength in vivo in humans. Journal of Sports Sciences, London, v. 27, n. 1, p. 59-68, 2009.

WEAKLEY, J. J. S.; TILL, K.; READ, D. B.; ROE, G. A. B.; DARRALL-JONES, J.; PHIBBS, P. J.; JONES, B. The effects of traditional, superset, and tri set resistance training structures on perceived intensity and physiological responses. European Journal of Applied Physiology, Berlin, v. 117, n. 9, p. 1877-89, 2017.

WILLARDSON, J. M.; BURKETT, L. N. A comparison of 3 different rest intervals on the exercise volume completed during a workout. The Journal of Strength \& Conditioning Research, Lincoln, v. 19, n. 1, p. 23-6, 2005.

\section{CONFLITO DE INTERESSE}

Os autores do estudo declaram não haver conflito de interesses.

\section{FINANCIAMENTO}

Este estudo não obteve nenhum apoio financeiro.

\section{ORCID E E-MAIL DOS AUTORES}

William Peneda Tozei

ORCID: 0000-0001-8056-9472.

E-mail:wtozei@gmail.com

Milton Pereira Amaral

ORCID: 0000-0002-0776-7111.

E-mail: milton92.ap@gmail.com

Washington Pires

ORCID: 0000-0002-8708-3245

E-mail: washpires@gmail.com

Renato Melo Ferreira

ORCID: 0000-0003-0944-6730.

E-mail: renato.mf@hotmail.com

Everton Rocha Soares (Autor Correspondente)

ORCID: 0000-0002-7765-5828.

E-mail: everton@ufop.edu.br 